\title{
BMJ Open Patient experience with discharge instructions in postdischarge recovery: a qualitative study
}

\author{
Molly J Horstman, ${ }^{1,2,3}$ Whitney L Mills, ${ }^{1,2}$ Levi I Herman, ${ }^{1,4}$ Cecilia Cai, ${ }^{5}$ \\ George Shelton, ${ }^{1,6}$ Tareq Qdaisat, ${ }^{7}$ David H Berger, ${ }^{1,8}$ Aanand D Naik ${ }^{1,2,3}$
}

To cite: Horstman MJ, Mills WL, Herman LI, et al. Patient experience with discharge instructions in postdischarge recovery: a qualitative study. BMJ Open 2017;7: 014842. doi:10.1136/bmjopen-2016014842

- Prepublication history and additional material is available. To view please visit the journal (http://dx.doi.org/ 10.1136/bmjopen-2016014842).

Received 20 0ctober 2016 Revised 12 January 2017 Accepted 16 January 2017

CrossMark

For numbered affiliations see end of article.

Correspondence to Dr Molly J Horstman; molly.horstman@bcm.edu

\section{ABSTRACT}

Objectives: We examined the role of discharge instructions in postoperative recovery for patients undergoing colorectal surgery and report themes related to patient perceptions of discharge instructions and postdischarge experience.

Design: Semistructured interviews were conducted as part of a formative evaluation of a Project ReEngineered Discharge intervention adapted for surgical patients.

Setting: Michael E. DeBakey VA Medical Center, a tertiary referral centre in Houston, Texas.

Participants: Twelve patients undergoing elective colorectal surgery. Interviews were conducted at the two-week postoperative appointment.

Results: Participants demonstrated understanding of the content in the discharge instructions. During the interviews, participants reported several positive roles for discharge instructions in their postdischarge care: a sense of security, a reminder of inhospital education, a living document and a source of empowerment. Despite these positive associations, participants reported that the instructions provided insufficient information to promote access to care that effectively addressed acute issues following discharge. Participants noted difficulty reaching providers after discharge, which resulted in the adoption of workarounds to overcome system barriers.

Conclusions: Despite concerted efforts to provide patient-centred instructions, the discharge instructions did not provide enough context to effectively guide postdischarge interactions with the healthcare system. Insufficient information on how to access and communicate with the most appropriate personnel in the healthcare system is an important barrier to patients receiving high-quality postdischarge care. Tools and strategies from team training programmes, such as team strategies and tools to enhance performance and patient safety, could be adapted to include patients and provide them with structured methods for communicating with healthcare providers post discharge.

\section{INTRODUCTION}

With the passage of the Affordable Care Act, the reduction of hospital readmissions for

\section{Strengths and limitations of this study}

- This study is one of the first to describe the role of written discharge instructions in postoperative recovery.

- Use of semi-structured interviews provided the opportunity to obtain detailed descriptions of the patient's postdischarge experience following elective colorectal surgery.

- Our study sample consisted mostly of older adult males with malignancy receiving care in a large, national integrated healthcare system, which may limit the generalisability of our findings.

medical and surgical patients has become a national priority. Although hospitals can control the quality of services provided to patients at the time of discharge, hospitals must rely on patients and caregivers to assume responsibility for the patient's medical care following discharge to avoid penalties related to excess readmissions. Patient education is a critical component of many readmission reduction interventions, yet patient understanding of the discharge plan at the time of discharge is often poor. ${ }^{1-6} \mathrm{~A}$ study of recently discharged patients aged 65 and older showed that $40 \%$ of patients were unable to accurately describe their admitting diagnosis and over $80 \%$ of patients had no understanding of medications stopped during their hospitalisation. ${ }^{78}$

Written instructions are provided to patients at discharge as a supplement to verbal inpatient education. The Joint Commission mandates hospitals to provide patients with written discharge instructions that are understandable to patients and their caregivers. ${ }^{9}$ However, the comprehension and role of discharge instructions in postdischarge recovery following surgery, especially after patients return home, remains unexplored. In a recent workshop from the Institute of Medicine, panelists advocated for additional research to explore patients' understanding of discharge 
instructions and to maximise the utility of discharge documents. ${ }^{10}$ Limited health literacy and cognitive impairment are well-established factors that limit patient and caregiver understanding of discharge instructions. ${ }^{6}$ Discharge instructions can be designed to overcome these limitations; however, simply offering patient-centred, lowliteracy instructions may be insufficient if the information provided is not actionable. ${ }^{10}{ }^{11}$ To maximise the impact of written instructions, discharge instructions must be able to support patient and caregiver activation for postdischarge self-care and empower them to become active members of their postdischarge care team. Furthermore, providers should be prepared to include patients and caregivers as part of a high-functioning care team. These instructions should facilitate communication among all the team members to reduce morbidity from complications or potential readmissions.

We examine the role of discharge instructions in postdischarge care for patients undergoing colorectal surgery and report themes related to patient perception of discharge instructions and postdischarge experience. This qualitative study was done as part of the Michael E. DeBakey VA Medical Center's efforts to adapt Project Re-Engineered Discharge (RED) to the specific needs of surgical patients. ${ }^{1}$

\section{METHODS}

\section{Participants and recruitment}

Interviews were conducted as part of a formative evaluation of the Project RED intervention adapted for colorectal surgical patients. Patients received an after hospital care plan (AHCP) tailored to postoperative care at the Michael E. DeBakey VA Medical Center. ${ }^{1}$ In the hospital, colorectal surgery patients are managed by one of two general surgery inpatient services, each with three residents and seven supervising attending surgeons. One nurse practitioner assists both teams. Review of discharge instructions is completed by the unit nurse immediately prior to patient discharge. The AHCP is a discharge tool that contains a patient-centric medication list, an appointment list with calendar and pharmacy information. The AHCP was designed to provide patients with clear contact information and instructions on how to seek medical care following discharge. Detailed process mapping with frontline staff identified the specific providers who were believed to be best able to respond to the postdischarge problems of colorectal surgery patients.

Our institution adapted the AHCP to include warning signs and symptoms for common problems faced by colorectal surgery patients after discharge. The warning signs and symptoms were developed based on a review of the literature and a Delphi expert consensus panel. ${ }^{12} 13$ The results of the Delphi panel were used to create a red, yellow and green colour scheme in which warning signs and symptoms that were red were emergencies that instructed patients to call 911 or go to the emergency room and warning signs and symptoms that were yellow directed patients to immediately call a medical provider for triage. For yellow symptoms, patients were instructed to call the surgery clinic Monday-Friday during business hours to speak to the surgery clerk or clinic nurse who would contact the physician assistant for triage support or to call the 24-hour nurse telephone triage service after hours and on weekends.

Recruitment and data collection occurred between June 2014 and January 2015 using purposeful criterion sampling. ${ }^{14}$ Clinicians identified potential participants from all patients scheduled in the preoperative surgery clinic. A chart review was conducted to identify patients who met eligibility criteria. Patients were eligible to participate if they underwent elective colorectal surgery for any reason during the study period and did not have cognitive impairment. Colorectal surgery was chosen for the intervention as it has one of the highest unplanned readmission rates nationally among surgical procedures. ${ }^{13}$ Patients were approached during their preoperative surgery clinic appointment to undergo informed consent.

\section{Data collection}

After obtaining written informed consent at the preoperative appointment, interviews were conducted with participants and caregivers, if present, at the 2-week postoperative follow-up appointment. All interviews were audio recorded and transcribed verbatim, and conducted by non-clinical research staff with no role in the care of the participants. Interviews were conducted by either a postdoctoral fellow with a $\mathrm{PhD}$ in Psychology or bachelor-level, experienced research assistants with training in qualitative interviewing. All interviewers were men. The mean interview length was $39 \mathrm{~min}$ (range 17$53 \mathrm{~min}$ ). Participants were told at the beginning of the interview that they would be asked a series of questions about their discharge paperwork and that the research staff was part of a team working to improve the process of patient discharge.

Interviews followed a semistructured interview guide that elicited patient understanding of the content and clarity of the AHCP (see online supplementary material). Participants were first asked about their experience using the AHCP after discharge including: 'Did you use the AHCP while you were at home?' and 'Did you call any of the phone numbers in the AHCP while you were at home?'. Probing questions were used as needed to clarify participant responses. Participants were asked to describe the information on each page and to describe how they would explain the information to a friend or family member ('Without looking at this care plan, imagine you were going to explain what this was to a friend or family member. What would you tell them?'). For every page of the AHCP, participants were asked questions to elicit their opinion about the information, such as: 'What would you change about this page to help other patients better understand the 
information on it?'. Patients also responded to questions about their general experience using the document during their recovery. For example, participants were asked to respond to the statement: 'Is there anything that you've learned through your experience that you would want to add or tell other patients about?"

\section{Qualitative analysis}

Our qualitative approach is based on the principles of thematic analysis because it used a deductive approach based on a priori templates for the content of the AHCP and an inductive approach arising from our emergent data to identify additional themes regarding patient experience following discharge. ${ }^{15}$ This combined approach allowed us to identify themes directly related to patient understanding of the specific content included in the AHCP and to identify themes related to the patient postdischarge experience that could not be defined a priori. One coinvestigator developed an initial coding framework, which was refined by the analysis team (see online supplementary material); two team members read the transcripts multiple times and independently made notations to code each interview and six team members met regularly to refine the coding scheme and code definitions. Discrepancies between coders were resolved during team meetings and team consensus was used to determine the final codes for the transcripts. Throughout the process and once coding was completed, team members discussed themes regarding patient postdischarge experience that emerged across interviews. All study authors reviewed the results of the analysis.

\section{RESULTS}

Of 28 patients who were eligible to participate and underwent surgery during the study period, 23 provided informed consent for the interviews. Of the 11 patients who were consented, but not interviewed, 3 did not have postoperative follow-up appointments, 1 was unable to answer questions at the postoperative follow-up appointment, 5 declined to be interviewed and 2 had appointments after thematic saturation was reached. Twelve patients completed interviews at the postoperative follow-up appointment. The mean age was 65 years (median 67.5 years, range 34-81) and nine participants were white $(75 \%)$ and three participants were African-American (25\%). One participant was female. Five participants were married. Most participants $(n=10)$ reported completing high school and of those, eight reported completing at least some education beyond high school. Eleven of the participants had a gastrointestinal malignancy and six of the participants received ostomies as part of their operation. Type of surgery varied with the most common surgeries being laparoscopic hemicolectomy $(n=4)$, open sigmoid colectomy $(n=2)$ and low anterior resection $(n=2)$. Mean length of stay was 5.8 days (SD 2.5 days).

\section{Clarity of written instructions}

All participants commented on the clarity of the discharge instructions. The instructions were described as 'self-explanatory' (Participant 3) and 'to the point and using words that are easy to understand' (Participant 7). Participants demonstrated comprehension of the content of the instructions and were able to provide detailed descriptions of the medication list, medication instructions, directions for how to refill medications, activity restrictions, warning sign and symptoms and future appointments. Specifically, participants were able to identify appropriate actions (eg, going to the emergency room or calling a medical provider) for specific warning signs and symptoms. The information provided in the instructions was felt to be complete providing 'all the information I would need' (Participant 5) for postdischarge recovery.

\section{AHCP design and layout}

Participants described the design and layout of the AHCP as an improvement from current discharge instructions provided by the electronic medical record used in the Veterans Health Administration, which are black and white and printed with a small serif font. One participant comparing the appointment list in the AHCP and the standard appointment list commented 'it's [the AHCP] better than the appointment deal they give you' (Participant 2). Participants appreciated the large font: 'It's not real small print where you've got to get the magnifying glass' (Participant 7). Overall, the information in the AHCP was felt to provide an appropriate amount of information with a layout that made it easy to find needed information. As one participant commented, 'It's laid out. It's not jumbled up. It's all laid out in a precise order' (Participant 7).

\section{Positive role of discharge instructions}

During the interviews, participants commented on the positive role of discharge instructions in their postdischarge care (table 1). Several participants spoke of discharge instructions as providing a sense of security. Participants compared the discharge instructions with a 'fire extinguisher' (Participant 10) and several participants reported that the instructions removed 'doubt' (Participant 2) and 'mental conflict' (Participant 7) in their postdischarge recovery. Participants also recognised the role of discharge instructions as a reminder of verbal education provided in the hospital. Participants cited the importance of a written reminder due to difficulty retaining verbal instructions because of medical acuity at time of discharge and the passage of time since discharge. In addition, participants discussed the role of discharge instructions as a living document. Ten of the participants commented on the ability to add information to the document and use the document as a tool to communicate questions with providers during follow-up. Finally, participants described discharge instructions as empowering them to take charge of their medical care. 
Table 1 Positive associations with discharge instructions

\begin{tabular}{|c|c|}
\hline Themes & Representative quote \\
\hline Security & $\begin{array}{l}\text {...the [phone] numbers, how can I put it? It's almost like knowing you've got a fire extinguisher. } \\
\text { (Participant 10) } \\
\text { It just removes a lot of mental conflict.(Participant 7) } \\
\text { I can tell you that if I was incapacitated where I couldn't tell them [family] anything, they could see for } \\
\text { themselves.(Participant 10) }\end{array}$ \\
\hline Reminder & $\begin{array}{l}\text { I was about to sit down in that tub and, of course, her way of making a point was to bring this [discharge } \\
\text { instructions] to me. She says, "What do you think you're doing?"... And she says, "You can't take a } \\
\text { bath. You have to take a shower."(Participant 10) } \\
\text { It's like an appointment map(Participant 4) } \\
\text {... it's got everything in here that the doctors have probably already told me even though I'm kind of } \\
\text { drugged up or whatever. I can still go back and recall it.(Participant 1) } \\
\text { It's all the instructions that, you know, they give you verbally before you leave...it's nice to have it written } \\
\text { down. Heh, like by now I've been on a weight restriction for so long I forgot I even had it.(Participant 11) }\end{array}$ \\
\hline $\begin{array}{l}\text { Living } \\
\text { Document }\end{array}$ & $\begin{array}{l}\text { Anything that comes up that's not already printed up...I can write it on there and fill in what's there. } \\
\text { (Participant 7) } \\
\text {...at least there is some room. In fact I noticed this on several pages. There is room and you can see } \\
\text { where if-if it came to where I needed to make notes; I had room to make notes. And-and I like that } \\
\text { idea.(Participant 10) } \\
\text { I was just tickled by the fact that uh I had another appointment come up. And I had room to put it there. } \\
\text { So, and as you can see, I utilized that. And I'm-and I'm grateful for that.(Participant 10) }\end{array}$ \\
\hline Empowerment & $\begin{array}{l}\text { That's right and if you don't follow this plan you're going to wind up back here.(Participant 4) } \\
\text {...someone else was doing it for you and you felt that [they weren't] doing the right thing for you...you } \\
\text { had the instruction to follow anyway. So you can just [say], "I don't need you anymore; I can do this for } \\
\text { myself you know."(Participant 4) }\end{array}$ \\
\hline
\end{tabular}

\section{Limitations of discharge instructions}

Despite reporting positive associations with discharge instructions, participants commented on several areas for improvement (table 2). The majority of participants reported that the instructions provided insufficient information to effectively access timely and effective medical care following discharge. Participants noted a lack of specificity in appointment location and provider contact information. One participant commented that information regarding clinic location may have meaning to hospital employees, but did not have meaning for patients: '...it doesn't tell us anything [regarding clinic location] ...It should be reflecting us. Not the doctor...' (Participant 9). Additionally, participants reported difficulty communicating with providers following discharge. Delays in returning calls by the appropriate providers were reported by participants. Participants also reported negative experiences following discharge when healthcare providers did not appreciate the significance of their symptoms or acknowledged that a concern was expressed that required timely intervention. To overcome systems barriers to timely communication, participants identified workarounds that included calling multiple providers to address the same issue. Participants also reported confusion regarding who to contact to resolve problems due to the number of different providers involved in their care. Finally, participants noted that the discharge instructions did not have sufficient information to manage some common non- medical postdischarge problems, such as instructions on how to cancel or change an appointment.

\section{DISCUSSION}

In our examination of how patients interact with instructions following hospital discharge, participants understood the content in the discharge instructions and reported several positive associations that have not previously been described. Discharge instructions offered patients a sense of security, served as a reminder for inhospital education, had the potential to be a living document and provided a source of empowerment for patients. Despite these positive associations, participants identified multiple concerns regarding the content of the information provided to access the healthcare system.

Several studies have focused on the quality of discharge summaries used for asynchronous communication between providers; yet, little attention has been paid to maximising patient discharge instructions to improve patients' postdischarge care. ${ }^{1016} 17$ Patients and caregivers must assume significant responsibility for medical care following discharge, which requires that patients and caregivers receive adequate preparation and training. For surgical patients, this education may be particularly important as the majority of surgical readmissions are for predictable complications related to surgery which may be amenable to outpatient 
Table 2 Limitations of discharge instructions in postdischarge experience with accessing healthcare system

Shemes

Experience with healthcare system

Additional contact information for common postdischarge problems

Communication workarounds

\section{Representative quote}

- And what l'd do to add to that would be the elevator to take and the room number....directions, the location.(Participant 5)

- ...they ought to put something down there telling you who you're calling. I mean there's so many 800 numbers out there nowadays.(Participant 6 )

- Well it ought to give me a list of doctors and nurses for patient care, names of the doctors, and maybe the phone numbers....and their extensions. (Participant 6)

- this has...all the information except for where. Right, they should have, you know, cancer center or whatever...I have no idea where this place is. (Participant 11)

- ... you get the runaround on the phone when you call them numbers. (Participant 6)

- ...that was kind of confusing. I didn't actually know which doctor was my doctor. Which actually did the surgery because there was about three people. I think it was a team of surgeons. And I don't-I didn't know which one of them was the head honcho to call.(Participant 12)

- We called; my wife called. She said she called and they said they would have to get back. There were three people that may call me back but we just went ahead and went on to the hospital because I was scared with all that blood.(Participant 12)

- Yeah well look l've got these appointments...l'm supposed to make these appointments. You know and if something comes up and I can't make it then you know it should be a number on here to call...So you know say okay, my driver can't come now...you know schedule me next week. (Participant 4)

- [in regards to what pharmacy to call for medication issues] whether to call the VA in Houston or in Alexandria...that would be needed to be there. (Participant 7)

- In my telephone directory, I have about eight listings for VA Houston...I have one or two of them [clinic phone numbers] there...eventually, I can get a warm body to talk to.(Participant 5)

- The only difficulty l've had was when my prescriptions had run out...It's not easy to contact your primary care physician. I left messages saying that I needed her to adjust the prescription information and for her to please order for me the refill...But I didn't have any information right away. I then checked back with this... refill number and by phone they advised me that one prescription had been requested...So it happened and the system worked. But I didn't have information up front because I wasn't able to talk to the primary physician.(Participant 5) treatment if identified early. ${ }^{13}{ }^{18-20}$ Patients, however, may be unable to learn new information at discharge due to their medical condition and caregivers may be absent at the time inhospital education is provided, leaving the discharge instructions as potentially the only source of information to guide postdischarge care. ${ }^{21}$

Our qualitative study adds to the current literature by noting several positive associations that patients have with discharge instructions. However, despite concerted efforts from our institution to provide clear contact information, the discharge instructions did not include enough information to effectively guide postdischarge communication with the healthcare system. Insufficient information for accessing the healthcare system and difficulties with contacting providers following discharge are important barriers to patients being able to receive high-quality postdischarge care. $^{22}$ Placing a greater emphasis on discharge instructions as a tool (in conjunction with coordinated inpatient education) to improve patient capacity for self-care may improve postdischarge care and reduce readmissions, but it will only do so in a system with care teams that are able to respond to patient concerns in a consistent and timely manner. ${ }^{18} 23$ Patients need innovative methods that selectively integrate them into the communication flow of healthcare teams managing postdischarge care.

The provision of high-quality medical care following hospital discharge requires high functioning teams. Successful teams are successful because they share common knowledge, skills and attitudes regarding their roles and responsibilities as team members. ${ }^{24}$ Team training programmes, such as crew resource 
Table 3 Closed-loop communication in postdischarge surgical recovery

Step 1: Patient and caregivers receive training on the use of patient-centred warning signs and symptoms to monitor postsurgery condition at home.

Yellow Zone:

This is your warning zone.If you have any of these problems you should contact your medical team at:

Step 2: Patient or caregiver identifies a problem and uses the discharge instructions to identify the specific provider who is able to assist in resolving the problem.

- Your surgical wound is:

- red;

- leaking liquid, blood or pus and

- opening up or getting worse.

- Your belly is:

- swelling up and feels very firm;

- hurting and the pain medicines don't help

- Any of the following are true:

- Eating and drinking make you vomit.

- You notice dark urine or less urine.

- You can't eat or drink anything for 24 hours.

- You have no bowel movement or do not pass any gas for 24 hours.

- You have a fever over $101.5^{\circ} \mathrm{F}$.

\section{Step 3: Closed-loop communication}

1. Patient calls specific provider and uses structured language from the discharge instructions that describes the severity of the problem.

2. Provider uses the structured language to develop a treatment plan.

3. Patient confirms the proposed treatment plan and is able to accept or ask for an alternative plan.
'My wound is opening up. I am in the yellow zone.'

'Your wound is opening up. We would like to have your wound examined in the clinic today. Can you come in at 3:00 pm?'

'Yes. My daughter can drive me to the clinic this afternoon. I will be there at 15:00.' management and team strategies and tools to enhance performance and patient safety (TeamSTEPPS), have been used in operating rooms, emergency departments and labour and delivery units to improve teamwork and patient outcomes. ${ }^{25} 26$ Although the goal of team training is to provide safe patient care, the training programmes themselves have been designed for medical providers. Unlike care teams in acute care medical settings in which the patient may take a passive role, patients and caregivers are critical members of postdischarge care teams. Patients and caregivers take an active role in the care team's common goal of returning the patient to his or her baseline clinical status and reducing avoidable use of health services, such as hospital readmissions, through at-home monitoring and self-care. The critical role of patients and caregivers in postdischarge care requires that team training programmes be adapted to include patients and caregivers as part of the team and to train them on strategies to become active participants in interprofessional postdischarge care teams. ${ }^{25-27}$

Effective communication is a key skill for successful teams. Inadequate communication between providers or among providers and patients and caregivers is a root cause in over $60 \%$ of sentinel events in the Joint Commission Sentinel Event database. ${ }^{28}$ Improving the processes through which patients and caregivers communicate with the healthcare system will be an important step to improving postdischarge communication; however, it will be insufficient to overcome all of the communication challenges patients and caregivers face. Effective team communication will require that patients and caregivers also change how they communicate with providers. Use of structured communication is a key component of team training programmes. ${ }^{26}$ Structured communication tools, such as closed-loop communication which ensures that the concerns voiced by a patient are understood by their medical provider as the patient intended, may help patients and caregivers effectively communicate care needs to the healthcare system (table 3). In the context of the colorectal surgery patients, the ability for patients and caregivers to clearly communicate that they are experiencing warning signs and symptoms from the discharge instructions using language that is familiar to all involved parties and actionable by providers has not been assessed. The use of shared language (clinical lexicon) between patients and providers within the context of structured communication tools may enhance the utility of the discharge instructions and result in care that meets patients' postdischarge needs. ${ }^{22}$

This study was completed at a single Veterans Affairs hospital with 12 patients undergoing elective colorectal surgery. Although the sample size was small, it was purposeful and adequate to achieve thematic saturation. ${ }^{29}$ Our sample consisted mostly of older adult males with colorectal malignancy. Our facility is a referral centre for surgical oncology within the Veterans Health Administration for veterans residing in several states along the US Gulf Coast. Our status as a referral centre 
may account for the higher proportion of patients in our sample undergoing colorectal surgery for malignancy compared with patients undergoing colorectal surgery at other facilities in the USA. ${ }^{30}$ Although this impacts the generalisability of our results, older patients with malignancy are an important subgroup of surgical patients to examine given their increased risk for adverse postdischarge events, including unplanned readmissions. ${ }^{18} 32$ The rates of ostomy placement in our sample are similar to previously published studies. ${ }^{30} 33$

Our final sample included patients who receive primary care in Houston and primary care at other medical centres in our Veteran Integrated System Network. Although this is typical for patients being seen in our surgical oncology clinic, this may have contributed to the confusion participants experienced regarding which provider to contact for questions. Although the study population limits generalisability, the themes identified are relevant to patients in other large integrated healthcare systems in the USA. As alternative payment systems that emphasise care coordination (eg, accountable care organisations) continue to grow, we believe that the issues we identified will be applicable to a wide range of non-federal healthcare systems. Furthermore, the findings are within the context of our institutions efforts to implement Project RED and the AHCP, which is a state-of-the-art patient discharge tool used in several healthcare settings. ${ }^{1}{ }^{34} 35$

\section{CONCLUSION}

This qualitative study provides new insight into patient perceptions of discharge instructions in their postdischarge care. Written discharge instructions are an important part of the discharge process, but patient experience with and use of written instructions have not been sufficiently explored in the literature. ${ }^{10}$ The potential roles of discharge instructions to provide security, educate patients, organise medical care and encourage empowerment are lofty and additional work is necessary to ensure provided discharge instructions fulfil this potential. In an era of increasing pressure to reduce surgical readmissions and with many hospital-based interventions offering limited improvement in readmissions, discharge instructions that connect patients and caregivers to appropriate resources and support patient and caregiver communication with providers following discharge are critical to improving the patient postdischarge experience. ${ }^{36}$

\footnotetext{
Author affiliations

${ }^{1}$ Center for Innovations in Quality, Effectiveness, and Safety (IQuESt); Michael E. DeBakey VA Medical Center, Houston, Texas, USA

${ }^{2}$ Section of Health Services Research, Department of Medicine, Baylor College of Medicine, Houston, Texas, USA

${ }^{3}$ VA Quality Scholars Coordinating Center, IQuESt, Michael E. DeBakey VA Medical Center, Houston, Texas, USA

${ }^{4}$ Jesse H. Jones Graduate School of Business, Rice University, Houston, Texas, USA

${ }^{5}$ Internal Medicine Residency Program, Baylor College of Medicine, Houston, Texas, USA
}

${ }^{6}$ Department of Family and Community Medicine, Baylor College of Medicine, Houston, Texas, USA

${ }^{7}$ Department of Pathology and Immunology, Baylor College of Medicine, Houston, Texas, USA

${ }^{8}$ Michael E. DeBakey Department of Surgery, Baylor College of Medicine, Houston, Texas, USA

Acknowledgements The authors thank Chris Corio from Engineered Care for his technical support. The authors would also like to thank Bryan

A. Campbell, PhD, for his assistance with developing the interview guide and conducting interviews.

Contributors WLM, DHB and ADN made substantial contributions to the conception or design of the work; MJH, WLM, LIH, CC, GS, TQ and ADN made substantial contributions to the acquisition, analysis or interpretation of the data; MJH, WLM and ADN drafted the manuscript; LIH, CC, GS, TQ and DHB revised the manuscript critically for important intellectual content; all authors gave the final approval of the version to be published.

Funding This research was supported by Health Services Research and Development Quality Enhancement Research Initiative (RRP 12-532) grant awarded to Dr Naik, and in part with resources at the Veterans Affairs HSR\&D Center for Innovations in Quality, Effectiveness and Safety (CIN 13-413) at the Michael E. DeBakey VA Medical Center, Houston, TX. Dr Horstman is supported by the VA Office of Academic Affiliations Advanced Fellowship in Health Services Research at IQuESt.

Disclaimer Engineered Care was not involved in the design of this study, analysis of the interviews or writing of the manuscript. Engineered Care did not provide any financial support for this study.

Competing interests none declared.

Ethics approval This study (IRB: H-29099) was reviewed and approved by the Institutional Review Board at Baylor College of Medicine and the VA Research and Development Committee at the Michael E. DeBakey VA Medical Centre.

Provenance and peer review Not commissioned; externally peer reviewed.

Data sharing statement Participants did not provide consent for the transcripts to be released outside of the Michael E. DeBakey VA Medical Center.

Open Access This is an Open Access article distributed in accordance with the Creative Commons Attribution Non Commercial (CC BY-NC 4.0) license, which permits others to distribute, remix, adapt, build upon this work noncommercially, and license their derivative works on different terms, provided the original work is properly cited and the use is non-commercial. See: http:// creativecommons.org/licenses/by-nc/4.0/

\section{REFERENCES}

1. Jack BW, Chetty VK, Anthony D, et al. A reengineered hospital discharge program to decrease rehospitalization: a randomized trial. Ann Intern Med 2009;150:178-87.

2. Coleman EA, Parry C, Chalmers $\mathrm{S}$, et al. The care transitions intervention: results of a randomized controlled trial. Arch Intern Med 2006;166:1822-8.

3. Makaryus AN, Friedman EA. Patients' understanding of their treatment plans and diagnosis at discharge. Mayo Clin Proc 2005;80:991-4.

4. Olson DP, Windish DM. Communication discrepancies between physicians and hospitalized patients. Arch Intern Med 2010;170:1302-7.

5. Albrecht JS, Gruber-Baldini AL, Hirshon JM, et al. Hospital discharge instructions: comprehension and compliance among older adults. J Gen Intern Med 2014;29:1491-8.

6. Coleman EA, Chugh A, Williams MV, et al. Understanding and execution of discharge instructions. Am J Med Qual 2013;28:383-91.

7. Horwitz LI, Moriarty JP, Chen C, et al. Quality of discharge practices and patient understanding at an academic medical center. JAMA Intern Med 2013;173:1715-22. 
8. Ziaeian B, Araujo KL, Van Ness PH, et al. Medication reconciliation accuracy and patient understanding of intended medication changes on hospital discharge. J Gen Intern Med 2012;27:1513-20.

9. Joint Commission on Accreditation of Healthcare Organizations. Comprehensive Accreditation and Certification Manual. Joint Commission Resources. 2015.

10. Roundtable on health literacy. Facilitating patient understanding of discharge instructions: workshop summary. Wasington DC: National Academies Press (US); 2014.

11. Chugh A, Williams MV, Grigsby J, et al. Better transitions: improving comprehension of discharge instructions. Front Health Serv Manage 2009;25:11-32.

12. Li LT, Mills WL, Gutierrez AM, et al. A patient-centered early warning system to prevent readmission after colorectal surgery: a national consensus using the delphi method. J Am Coll Surg 2013;216:210-16.

13. Li LT, Mills WL, White DL, et al. Causes and prevalence of unplanned readmissions after colorectal surgery: a systematic review and meta-analysis. J Am Geriatr Soc 2013;61: 1175-81.

14. Palinkas LA, Horwitz SM, Green CA, et al. Purposeful sampling for qualitative data collection and analysis in mixed method implementation research. Adm Policy Ment Health 2015;42:533-44.

15. Fereday J, Muir-Cochrane E. Demonstrating rigor using thematic analysis: a hybrid approach of inductive and deductive coding and theme development. Int J Qual Methods 2006;5: 80-92.

16. Kripalani S, LeFevre F, Phillips $\mathrm{CO}$, et al. Deficits in communication and information transfer between hospital-based and primary care physicians: implications for patient safety and continuity of care. JAMA 2007;297:831-41.

17. Horwitz LI, Jenq GY, Brewster UC, et al. Comprehensive quality of discharge summaries at an academic medical center. $J$ Hosp Med 2013;8:436-43.

18. Merkow RP, Ju MH, Chung JW, et al. Underlying reasons associated with hospital readmission following surgery in the United States. JAMA 2015;313:483-95.

19. Hinami K, Bilimoria KY, Kallas PG, et al. Patient experiences after hospitalizations for elective surgery. Am J Surg 2014;207:855-62.

20. Kable A, Gibberd R, Spigelman A. Complications after discharge for surgical patients. ANZ J Surg 2004;74:92-7.

21. Cain $\mathrm{CH}$, Neuwirth $\mathrm{E}$, Bellows $\mathrm{J}$, et al. Patient experiences of transitioning from hospital to home: an ethnographic quality improvement project. J Hosp Med 2012;7:382-7.
22. Horstman MJ, Stewart DE, Naik AD. Improving patients postdischarge communication: making every word count. Circulation 2014;130:1091-4.

23. Leppin AL, Gionfriddo MR, Kessler M, et al. Preventing 30-day hospital readmissions: a systematic review and meta-analysis of randomized trials. JAMA Intern Med 2014;174:1095-107.

24. Baker DP, Salas E, King $\mathrm{H}$, et al. The role of teamwork in the professional education of physicians: current status and assessment recommendations. Jt Comm J Qual Patient Saf 2005;31:185-202.

25. Weaver SJ, Dy SM, Rosen MA. Team-training in healthcare: a narrative synthesis of the literature. BMJ Qual Saf 2014;23:359-72.

26. King HB, Battles J, Baker DP, et al. TeamSTEPPS: team strategie and tools to enhance performance and patient safety. 2008. http:// www.ahrq.gov/downloads/pub/advances2/vol3/Advances-King_1.pdf (accessed 17 Mar 2016).

27. Baker DP, Gustafson S, Beaubien JM, et al. Medical team training programs in health care. 2005. http://www.ncbi.nlm.nih.gov/books/ NBK20580/pdf/Bookshelf_NBK20580.pdf (accessed 17 Mar 2016).

28. The Joint Commission. Improving America's hospitals: the joint commission's annual report on quality and safety. 2007. http://www. jointcommission.org/assets/1/6/2007_Annual_Report.pdf (accessed 10 Mar 2016)

29. Guest G, Bunce A, Johnson L. How many interviews is enough? An experiment with data saturation and variability. Field Methods 2006;18:59-82.

30. Wick EC, Shore AD, Hirose K et al. Readmission rates and cost following colorectal surgery. Dis Colon Rectum 2011;54:1475-9.

31. Abarca F, Saclarides TJ, Brand MI. Laparoscopic colectomy: complications causting reoperation or emergency room/hospital readmissions. Am Surg 2011;77:65-9.

32. Orcutt ST, Li LT, Balentine CJ, et al. Ninety-day readmission after colorectal cancer surgery in a Veterans Affairs cohort. J Surg Res 2016;201:370-7.

33. Kiran RP, Delaney CP, Senagore AJ, et al. Outcomes and prediction of hospital readmission after intestinal surgery. J Am Coll Surg 2004; 198:877-83.

34. Goldman LE, Sarkar U, Kessell E, et al. Support from hospital to home for elders: a randomized trial. Ann Intern Med 2014; 161:472-81.

35. Adams CJ, Stephens K, Whiteman K, et al. Implementation of the Re-Engineered Discharge (RED) toolkit to decrease all-cause readmission rates at a rural community hospital. Qual Manag Health Care 2014;23:169-77.

36. Rennke S, Nguyen OK, Shoeb MH, et al. Hospital-initiated transitional care interventions as a patient safety strategy: a systematic review. Ann Intern Med 2013;158(Pt 2):433-40. 